\title{
Tabagismo e Externalidades da Alfabetização no Ceará
}

\author{
Felipe Garcia Ribeiro ${ }^{1}$ \\ André Carraro $^{2}$
}

Resumo: Este trabalho verifica se a presença de indivíduos alfabetizados em domicílios que residem indivíduos analfabetos é capaz de reduzir o hábito de tabagismo dos analfabetos no estado brasileiro do Ceará. Com informações da pesquisa especial da PNAD 2008 sobre tabagismo, a utilização do método de variáveis instrumentais encontrou evidências de que pessoas analfabetas que convivem com outras alfabetizadas tem em média uma menor probabilidade (variando de -9,5p.p a -7,op.p) de ser fumante do que analfabetos que não convivem com pessoas alfabetizadas. Estes resultados são robustos às diferentes definições de alfabetização adotadas neste trabalho.

Palavras-Chave: Tabagismo, Externalidades da Alfabetização, Analfabetos NãoIsolados.

Classificação JEL: I15; I18; I30.

1 Universidade Federal de Pelotas (UFPEL). Programa de Pós-Graduação em Organizações e Mercados (PPGOM/UFPEL). E-mail: felipe.garcia.rs@gmail.com

2 Universidade Federal de Pelotas (UFPEL). Programa de Pós-Graduação em Organizações e Mercados (PPGOM/UFPEL). E-mail: andre.carraro@gmail.com 


\title{
Smoking and Literacy Externalities: evidence from Ceará
}

\begin{abstract}
This paper investigates whether the presence of literate individuals in the household reduce the likelihood of smoking among illiterate individuals who share that same household in Brazilian state, Ceará. Based on the information of the PNAD 2008's research about smoking habits, the use of the instrumental variable's method showed that illiterate individuals that live among literate people have, on average, a smaller probability (varying between-9.op.p and-7.op.p) of smoking than illiterate individuals who do not live with literate people. These results are robust to the different definitions of literacy adopted.
\end{abstract}

Key Words: Smoking, Literacy Externalities, Proximate Literate.

JEL Classification: I15; I18; I30.

\section{Introdução}

É bem documentado na literatura de desenvolvimento econômico que a alfabetização acarreta, privadamente, em melhores salários e melhor qualidade de vida (Blunch e Verner (2000), Green e Riddell (2003), Chiswick e Repetto (2000) e De Baldini Rocha e Ponczeck (2011)). No entanto, os benefícios da alfabetização podem extrapolar os ganhos privados e gerar externalidades positivas para pessoas analfabetas. Basu e Foster (1998) levantam a possibilidade de que indivíduos analfabetos que convivem com pessoas alfabetizadas estão em uma situação, em termos de acesso à informação, bem diferente daqueles analfabetos que estão isolados do convívio de pessoas alfabetizadas. O ponto central desses autores é que a alfabetização pode ser pensada como um bem público dentro de um domicílio.

Para exemplificar, suponha uma campanha de saúde pública de combate ao tabagismo baseada na distribuição de panfletos em postos de saúde localizados em zonas rurais. Indivíduos analfabetos que estão em contato com pessoas alfabetizadas podem ter acesso às informações oferecidas pelo governo se os alfabetizados que os circundam lerem, ou explicarem os malefícios à saúde gerados pelo consumo de tabaco. Já indivíduos analfabetos isolados de pessoas alfabetizadas não têm acesso a essas mesmas informações. Claramente, 
o grupo de pessoas analfabetas que tem contato com pessoas alfabetizadas (daqui em diante Analfabetos Não-Isolados, $A N I$ ) está em uma situação, em termos de bem-estar, ou qualidade de vida, superior ao grupo de analfabetos sem contato com pessoas alfabetizadas (daqui em diante analfabetos isolados, $A I$ ).

O presente estudo busca identificar se a convivência com alguma pessoa alfabetizada dentro da unidade domiciliar exerce algum impacto negativo sobre a probabilidade de um indivíduo analfabeto ser fumante. Para tanto, utilizam-se as informações para um Estado do Brasil, Ceará, presentes na Pesquisa Nacional por Amostra de Domicílios (PNAD) 2008, que contém uma pesquisa especial sobre consumo de tabaco. A PNAD levanta anualmente informações referentes ao mercado de trabalho, educação e condições de habitação, além de eventuais pesquisas que abordam questões pertinentes a análise de política pública, como a pesquisa de 2008 sobre tabagismo.

A proposta do trabalho é importante para as discussões a respeito da relevância da educação sobre tabagismo, existência de externalidades da alfabetização, e orientação de políticas públicas de combate a pobreza e a saúde. Em relação à literatura que estuda educação e tabagismo este estudo contribui de duas formas. Primeiro, diferente da literatura internacional, que avalia o impacto privado da educação e da alfabetização sobre os hábitos de tabagismo, esse artigo se propõe a avaliar o impacto social da alfabetização. Isso é dizer que o trabalho avalia o impacto da alfabetização de um indivíduo para a escolha do tabagismo em outro individuo analfabeto. Segundo, o trabalho avalia o impacto da alfabetização e, não da escolaridade. Ao se falar em alfabetização está se avaliando o impacto dos projetos sociais e das políticas públicas de redução do analfabetismo sobre as escolhas de saúde de outros indivíduos analfabetos.

Diante da existência de impactos positivos das externalidades da alfabetização na redução do tabagismo, formuladores de políticas públicas, tanto de saúde quanto de educação, têm mais um motivo para justificar o balizamento de recursos públicos para a alfabetização da população, além de contar com um instrumento para análise de custo e benefício das políticas. A partir da estimativa do impacto, é possível quantificar o retorno de uma política de alfabetização, para um dado orçamento, em termos de redução no número de analfabetos fumantes.

A escolha do Ceará para a realização desse estudo é justificada pelo fato de que este é um dos Estados com maior presença de analfabetos isolados e fumantes, o que será mostrado ao longo do texto. Ribeiro e Souza (2013) na investigação que fazem do perfil da alfabetização da população brasileira, com base nas definições de analfabetismo isolado, ratificam que são nos Estados do Nordeste que há a maior presença de analfabetos isolados, e portanto, o fenômeno da restrição total a informações e serviços que requerem o uso da escrita e da leitura deve ser mais presente nessa região. 
Em termos metodológicos, este trabalho se distingue totalmente dos já realizados na literatura de externalidades da alfabetização, uma vez que é o primeiro que se tem conhecimento a utilizar uma estratégia empírica cuja hipótese central para a identificação do impacto é de seleção em fatores não-observáveis. No entanto, na literatura que investiga educação e tabagismo estratégias empíricas alicerçadas no uso de variáveis instrumentais são usuais.

Para o cumprimento dos objetivos propostos, o trabalho está estruturado da seguinte forma: na próxima seção faz-se uma discussão detalhada da literatura de externalidades da alfabetização para contextualizar o presente trabalho no debate. Além disso, apresenta-se o perfil da alfabetização no Brasil em 2008 com as definições de $A I$ e $A N I$. Como já dito, será visto que o Ceará, assim como os demais Estados do Nordeste, concentra boa parte dos $A I$ do país, e que a participação da população de $A I$ dentro da população do Ceará é relativamente alta em relação ao resto do país.

A terceira seção discute a problemática do tabagismo no Brasil e no Mundo, discute alguns trabalhos que investigam a relação entre educação e consumo de tabaco e apresenta o perfil de tabagismo da população brasileira, dando destaque para o Estado do Ceará. Será visto que o Estado não ocupa uma boa posição no que tange os hábitos de tabagismo da sua população em relação aos demais estados.

A quarta seção apresenta a estratégia empírica do trabalho e a seleção amostral que é obtida da PNAD de 2008. Discute-se a validade das hipóteses subjacentes aos métodos adotados para identificação das externalidades, a saber: 0 modelo de probabilidade linear estimado por Mínimos Quadrados Ordinários (MQO), o modelo Probit e o Método de dois Estágios com Variáveis instrumentais. Utilizam-se variáveis instrumentais para o perfil de alfabetização dos domicílios em que residem analfabetos com a finalidade de se obter melhores estimativas do impacto causal das externalidades sobre a condição de consumo de tabaco dos analfabetos. Os instrumentos utilizados são obtidos a partir de mudanças exógenas de políticas educacionais ocorridas no Brasil. A quinta seção apresenta e discute os resultados obtidos. A sexta faz uma síntese do trabalho e recomendações de políticas públicas.

As estimativas obtidas pelo método de estimação em dois estágios com variáveis instrumentais apontam que indivíduos analfabetos que convivem com pessoas alfabetizadas têm em média uma probabilidade entre -9.5\% e -7.0\% de serem fumantes quando comparados a indivíduos analfabetos isolados do convívio com pessoas alfabetizadas. Os resultados são robustos às diferentes definições utilizadas para definir o status de alfabetização dos indivíduos. 


\section{Externalidades e Perfil de Alfabetização da População}

Após o trabalho seminal de Basu e Foster (1998) os trabalhos seguintes seguiram em duas trilhas não excludentes de pesquisa. Em uma delas a preocupação dos autores está em sofisticar a medida tradicional de alfabetização (a razão entre o total de alfabetizados e o total de pessoas), com a incorporação dos conceitos de externalidades, analfabetismo isolado e analfabetismo não-isolado para o diagnóstico de regiões em piores situações em termos de bem-estar. Destacam-se entre esses trabalhos Basu e Lee (2008), Dutta (2004), Mishra (2001), Mukherjee e Gupta (2006), Maddox (2007), Lee (2008), Subramanian (2004) e Valentini (2002).

Já na segunda trilha, na qual o presente estudo se enquadra, o objetivo dos pesquisadores é verificar empiricamente se existe de fato externalidades da alfabetização, e se essas podem ser capturadas no mercado de trabalho em termos de maiores salários (decorrentes de maior produtividade) ou em indicadores de saúde. Na literatura internacional, para o caso de análise no mercado de trabalho, os principais estudos são de Basu, Narayan e Ravallion (2001), Hojo (2004), Sarr (2004) e Iversen e Palmer-Jones (2008). Em geral, os três primeiros estudos acima apontam para um significativo prêmio de salário para os indivíduos ANI (em torno de 15.0\%), que pode ser ainda maior se os indivíduos alfabetizados forem mulheres. Já o estudo de Iversen e Palmer-Jones (2008) destaca a possibilidade de que os efeitos encontrados podem estar contaminados por problemas de seleção, e características não-observáveis tanto dos analfabetos quanto dos indivíduos alfabetizados. No Brasil, o trabalho de Ribeiro e Cechin (2012) identificou que pais analfabetos beneficiários do programa bolsa família que possuem filhos alfabetizados recebem em média 10,96\% a mais do que aqueles pais analfabetos que não possuem filhos alfabetizados.

Referente à mensuração de externalidades da alfabetização em indicadores de saúde (tais como altura e peso) podem ser citados os trabalhos de Gibson (2001), Almeyda-Duran (2005) e Borooah (2002). Os dois primeiros autores encontram associação positiva entre externalidades da alfabetização e indicadores de saúde das crianças, enquanto Borooah (2002) encontra evidências de que apenas a alfabetização per si exerce algum impacto na medida de peso de crianças.

Quanto ao perfil de alfabetização brasileira, em 2008, da população com 15 ou mais de idade, $10.0 \%$ não sabia ler e escrever. Deste número $28.7 \%$ era analfabeto isolado do convívio domiciliar com pessoas alfabetizadas, o que representa uma população de aproximadamente quatro milhões de pessoas totalmente privadas do acesso às informações e serviços que requerem o uso de leitura ou escrita. Embora esta população represente apenas 3.0\% do total do país, é um contingente de pessoas cuja população é maior do que a de muitos estados. 
A Tabela 1 abaixo apresenta a distribuição da população com 15 ou mais anos de idade nos Estados brasileiros por status de Alfabetização em 2008. Os estados estão ranqueados de acordo com a participação de $A I$ na população total deste grupo no país. O Ceará aparece como o quarto Estado brasileiro que mais tem $A I$, sendo que a sua população total é bem menor do que a dos estados que aparecem a sua frente neste ranking: Bahia, Minas Gerais e São Paulo (ver última coluna).

A elevada participação da população $A I$ do Ceará no total de $A I$ do país repercute negativamente no perfil de alfabetização da sua própria população: $5.4 \%$ das pessoas com mais de 15 anos de idade são $A I$, o que representa $28.1 \%$ do total dos analfabetos do estado. Este resultado coloca o Ceará como o sexto estado brasileiro com o maior número de $A I$ na própria população. 
TABELA 1: DISTRIBUIÇÃO DA POPULAÇÃO BRASILEIRA COM 15 OU MAIS ANOS DE IDADE ENTRE OS ESTADOS SEGUNDO O PERFIL DE ALFABETIZAÇÃO

\begin{tabular}{|c|c|c|c|c|c|}
\hline UF & $\begin{array}{l}\text { Alfabe- } \\
\text { tizados } \\
(\%)\end{array}$ & $\begin{array}{l}\text { Analfa- } \\
\text { betos (\%) }\end{array}$ & $\begin{array}{l}A I \\
(\%)\end{array}$ & $\begin{array}{l}A N I \\
(\%)\end{array}$ & $\begin{array}{l}\text { Total } \\
(\%)\end{array}$ \\
\hline Bahia & 6.9 & 13.0 & 14.9 & 12.2 & $7 \cdot 5$ \\
\hline Minas Gerais & 10.8 & $9 \cdot 3$ & 8.9 & 9.4 & 10.7 \\
\hline São Paulo & 23.6 & 10.6 & 8.5 & 11.4 & 22.3 \\
\hline Ceará & $3 \cdot 9$ & 8.3 & 8.3 & 8.3 & $4 \cdot 3$ \\
\hline Pernambuco & 4.0 & 7.9 & 8.1 & 7.9 & 4.4 \\
\hline Paraíba & 1.7 & 4.6 & 5.6 & 4.3 & 2.0 \\
\hline Maranhão & 2.7 & 5.9 & $5 \cdot 4$ & 6.1 & 3.0 \\
\hline Alagoas & 1.3 & 4.0 & $5 \cdot 3$ & 3.4 & 1.5 \\
\hline Piauí & 1.4 & 3.9 & $4 \cdot 3$ & 3.8 & 1.6 \\
\hline Rio de Janeiro & $9 \cdot 3$ & $3 \cdot 9$ & 3.6 & 4.0 & 8.7 \\
\hline Pará & 3.5 & 4.2 & 3.6 & 4.5 & 3.5 \\
\hline Paraná & 5.8 & $3 \cdot 7$ & 3.5 & 3.8 & 5.6 \\
\hline Goiás & 3.1 & 2.9 & 3.2 & 2.7 & 3.1 \\
\hline Rio Grande do Norte & 1.4 & 3.2 & 3.1 & $3 \cdot 3$ & 1.6 \\
\hline Rio Grande do Sul & 6.3 & 3.0 & 2.9 & 3.0 & $5 \cdot 9$ \\
\hline Sergipe & 1.0 & 1.7 & 1.9 & 1.7 & 1.0 \\
\hline Espírito Santo & 1.9 & 1.6 & 1.7 & 1.6 & 1.8 \\
\hline Mato Grosso & 1.6 & 1.5 & 1.5 & 1.5 & 1.6 \\
\hline Santa Catarina & 3.5 & 1.5 & 1.1 & 1.6 & $3 \cdot 3$ \\
\hline Tocantins & 0.6 & 0.9 & 1.0 & 0.9 & 0.6 \\
\hline Mato Grosso do Sul & 1.2 & 1.0 & 0.9 & 1.0 & 1.2 \\
\hline Amazonas & 1.6 & 1.3 & 0.9 & 1.5 & 1.6 \\
\hline Rondônia & 0.8 & 0.7 & 0.7 & 0.7 & 0.8 \\
\hline Acre & 0.3 & 0.4 & 0.5 & 0.4 & 0.3 \\
\hline Distrito Federal & 1.4 & 0.5 & 0.3 & 0.6 & 1.3 \\
\hline Roraima & 0.2 & 0.2 & 0.2 & 0.2 & 0.2 \\
\hline Amapá & 0.3 & 0.1 & 0.1 & 0.1 & 0.3 \\
\hline Brasil & 100 & 100 & 100 & 100 & 100 \\
\hline
\end{tabular}

Fonte: PNAD 2008 e elaboração Própria. 


\section{A Problemática do Tabaco e os Estudos da Importância da Educação}

Segundo a Organização Mundial da Saúde (WHO, 2002) o tabagismo é considerado o principal fator de risco para diversas doenças crônicas, fato que lhe atribui a responsabilidade por um elevado número de mortes prematuras no mundo. Considerado como uma epidemia para os pesquisadores da área de saúde pública, a cada ano o tabagismo é responsável pela perda de cerca de três milhões de vidas. A previsão para os próximos 30 anos indica que a epidemia tabágica será responsável por 10 milhões de mortes, sendo $70 \%$ delas concentradas em países em desenvolvimento ${ }^{1}$, que em geral são carentes de financiamento público para programas sociais.

No Brasil, as estimativas indicam 200 mil mortes ao ano causadas pelo hábito do tabagismo. Os estudos em epidemiologia (entre eles, Monteiro, 2007) associam o hábito de fumar com mais de 12 diferentes doenças, entre elas, o câncer de faringe, esôfago, estômago, laringe, pulmão, além de doenças cardiovasculares e cerebrovasculares. Todas essas doenças trazem um elevado custo econômico para o Sistema Único de Saúde (SUS). Segundo Pinto e Ugá (2010), para o ano de 2005, as internações para todas as patologias custaram $\mathrm{R} \$ 3.809 .446 .536,23$ ao SUS, sendo que 6,9\% foram custos atribuídos diretamente ao tabagismo, o equivalente a $\mathrm{R} \$ 262.965 .161,14$. Como consequência desses números, o mundo como um todo e, o Brasil especificamente, tem promovido programas sociais de prevenção à prática do tabagismo ${ }^{2}$, focado principalmente em escolas públicas, baseados na hipótese de que a orientação quanto aos malefícios e consequências do tabagismo possa ser uma importante ferramenta no desestímulo de fumar para os jovens e para com quem convive com eles.

Da população com 15 ou mais anos de idade no Brasil em 2008, 17,2\% fazia uso de tabaco fumado em frequência diária ou ocasional, o que define fumante corrente. Esta parcela representa aproximadamente 25 milhões de pessoas expostas diretamente a todos os malefícios que o uso de tabaco pode acarretar à saúde. O Ceará ocupa a quinta posição no ranking dos Estados com maior parcela de fumantes entre a população. Da população cearense na faixa etária da pesquisa, $19.4 \%$ das pessoas são fumantes (ver Tabela 2)

\footnotetext{
${ }^{1}$ Dados fornecidos por Roemer (1995) e WHO (1999).

${ }^{2} \mathrm{~A}$ maior parte desses programas é promovida pelas secretarias estaduais e municipais de saúde e educação, ver INCA (2011).
} 
TABELA 2: CONDIÇÃO DE USO DE TABACO FUMADO POR UNIDADES DA FEDERAÇÃO - PESSOAS COM 15 OU MAIS ANOS DE IDADE (MIL PESSOAS)

\begin{tabular}{|c|c|c|c|c|c|}
\hline UF & $\begin{array}{l}\text { Fuman- } \\
\text { tes }\end{array}$ & $\begin{array}{l}\text { Não Fu- } \\
\text { mantes }\end{array}$ & Total & $\begin{array}{l}\text { Fuman- } \\
\text { tes (\%) }\end{array}$ & $\begin{array}{l}\text { Tot a l } \\
(\%)\end{array}$ \\
\hline Acre & 101 & 357 & 458 & 22.1 & $7 \cdot 5$ \\
\hline Rio Grande do Sul & 1759 & 6718 & 8477 & 20.7 & 10.7 \\
\hline Paraíba & 568 & 2239 & 2807 & 20.2 & 22.3 \\
\hline Piauí & 458 & 1852 & 2310 & 19.8 & $4 \cdot 3$ \\
\hline Ceará & 1200 & 4997 & 6197 & 19.4 & $4 \cdot 4$ \\
\hline Tocantins & 174 & 753 & 926 & 18.7 & 2.0 \\
\hline $\begin{array}{l}\text { Mato Grosso do } \\
\text { Sul }\end{array}$ & 324 & 1424 & 1748 & 18.5 & 3.0 \\
\hline Paraná & 1483 & 6576 & 8059 & 18.4 & 1.5 \\
\hline Roraima & 50 & 230 & 281 & 17.9 & 1.6 \\
\hline Pará & 902 & 4156 & 5058 & 17.8 & 8.7 \\
\hline Espírito Santo & 467 & 2151 & 2618 & 17.8 & $3 \cdot 5$ \\
\hline Pernambuco & 1117 & 5218 & 6335 & 17.6 & 5.6 \\
\hline Minas Gerais & 2683 & 12586 & 15270 & 17.6 & 3.1 \\
\hline Mato Grosso & 383 & 1857 & 2240 & 17.1 & 1.6 \\
\hline Santa Catarina & 809 & 3925 & 4735 & 17.1 & $5 \cdot 9$ \\
\hline Goiás & 744 & 3633 & 4377 & 17.0 & 1.0 \\
\hline $\begin{array}{l}\text { Rio Grande do } \\
\text { Norte }\end{array}$ & 392 & 1924 & 2316 & 16.9 & 1.8 \\
\hline São Paulo & 5344 & 26567 & 31911 & 16.7 & 1.6 \\
\hline Maranhão & 702 & 3621 & 4324 & 16.2 & $3 \cdot 3$ \\
\hline Alagoas & 347 & 1845 & 2191 & 15.8 & 0.6 \\
\hline Bahia & 1651 & 9034 & 10684 & $15 \cdot 4$ & 1.2 \\
\hline Rio de Janeiro & 1906 & 10600 & 12506 & 15.2 & 1.6 \\
\hline Rondônia & 163 & 929 & 1091 & 14.9 & 0.8 \\
\hline Amapá & 59 & 365 & 425 & 14.0 & 0.3 \\
\hline Amazonas & 317 & 1959 & 2277 & 13.9 & 1.3 \\
\hline Distrito Federal & 254 & 1649 & 1903 & 13.4 & 0.2 \\
\hline Sergipe & 194 & 1281 & 1474 & 13.1 & 0.3 \\
\hline Brasil & 24552 & 118446 & 142999 & 17.2 & 100 \\
\hline
\end{tabular}

Fonte: PNAD 2008. 
Assim como a alfabetização, a educação em seu contexto mais geral está fortemente documentada como um fator determinante na melhoria em variáveis econômicas e de bem-estar. Contudo, além dos ganhos individuais, a educação pode afetar uma série de resultados sociais como escolhas relacionadas com o casamento, com a fertilidade, e com os cuidados à saúde (Grossman, 2006). Dentro do grupo de trabalhos que relacionam educação com cuidados à saúde têm recebido destaque nos últimos anos os que estimam o impacto da educação sobre a decisão de fumar (Tenn, Herman e Wendling, 2010; Elité e Jones, 2011; Kemptner, Jürges e Reinhold, 2011). Esses trabalhos têm por objetivo avaliar o impacto da educação como ferramenta de combate ao tabagismo baseados em três argumentos econômicos (Grossman, 2000).

O primeiro é a chamada hipótese de eficiência educacional, a qual argumenta que entre indivíduos com maior escolaridade, a proporção de fumantes tende a ser menor devido ao maior conhecimento e acesso à oferta de informação sobre os riscos à saúde que o tabagismo oferece. O segundo argumento econômico é a chamada hipótese de eficiência locativa. O que sustenta essa hipótese é o pressuposto que indivíduos com maior capital humano são mais capazes de avaliar o "valor da vida", ou o valor da atividade econômica desenvolvida. Se indivíduos com maior escolaridade ganham salários maiores, então, a escolha por fumar não só aumenta o risco de produzir doenças como aumenta a probabilidade de perdas econômicas no futuro, gerando (ou aumentando) o custo de oportunidade pela escolha em ser fumante. O terceiro argumento baseado em Lange (2011) é de que indivíduos com maior escolaridade acreditam mais nos resultados de pesquisas científicas dos riscos do tabagismo e menos em crenças populares.

Apesar da consistência teórica desses argumentos que justificam a existência de uma relação causal entre educação e escolha pelo tabagismo, empiricamente persiste o problema de que fatores não-observáveis podem confundir o efeito causal da educação sobre a escolha pelo tabagismo. Farrel e Fuchs (1982) foram pioneiros em argumentar que a associação pode ser espúria pela existência de uma terceira variável, como a presença de indivíduos com baixas taxas de desconto do tempo ou com maiores habilidades cognitivas, que afeta a demanda por educação, reduzindo a chance de o indivíduo ser fumante. O argumento básico desenvolvido em Farrel and Fuchs (1982) e, depois ampliado em Fuchs (2004) e em Cutler and Lleras-Muney (2006) é que o nível de escolaridade aumenta a importância que o indivíduo atribui para os eventos no futuro. Se escolaridade afeta o "valor da vida" no futuro, então, é provável que o indivíduo esteja propenso a investir mais em saúde, reduzindo a escolha pelo tabagismo, dada a aquisição de um ano a mais de escolaridade.

Baseados no alerta dado por Farrel e Fuchs (1982) de que a simples e direta mensuração do impacto da educação sobre a escolha pelo tabagismo poderia estar gerando resultados superestimados, os recentes trabalhos da área de 
pesquisa em economia da saúde buscam identificar o impacto causal por meio da estratégia de identificação de variáveis instrumentais, o que reduz o problema de endogeneidade existente. No entanto, essa estratégia só é válida quando as variáveis instrumentais são correlacionadas com a educação, mas não correlacionadas com características não observáveis que afetam a saúde do indivíduo. Neste contexto, as recentes pesquisas na área exploram reformas em políticas educacionais, que influenciam o nível de escolaridade do indivíduo sem estar correlacionadas com demais fatores não observáveis. A principal vantagem desses instrumentos está no fato de não existir motivo pelo qual a reforma na educação afete as escolhas individuais pelo tabagismo que não seja pela própria educação.

Seguindo essa estratégia Elité e Jones (2011) avaliam o impacto da educação sobre a escolha pelo tabagismo para a França, utilizando como variável instrumental a ampliação da oferta de escolas ocorrida em consequência de diversas reformas no sistema de ensino francês que alteraram a estrutura curricular de uma geração para outra no período pós-segunda guerra mundial. Seus resultados apontam para uma redução média de 1,51\% na escolha pelo tabagismo para as mulheres e, de $2,77 \%$ para os homens, dado um ano a mais de escolaridade. Kemptner, Jürges e Reinhold (2011) analisando o caso da Alemanha adotaram a mesma estratégia de utilizar uma reforma do ensino. Nesse caso, foi utilizada a reforma curricular ocorrida entre os anos de 1949 e 1969, que substituiu a exigência de 8 para 9 anos para o ensino básico. $O$ trabalho conclui que a escolha pelo tabagismo é afetada negativamente pela escolaridade, de forma mais intensa para os homens do que para as mulheres. Para o caso da Alemanha, Kemptner, Jürges e Reinhold (2011) relatam que um ano a mais de estudo reduz a escolha pelo tabagismo em 2,6\% para os homens e, 0,5\% para as mulheres, por meio de regressões utilizando Mínimos Quadrados Ordinários. Quando utilizado o método de variáveis instrumentais as estimativas não sugerem a existência de qualquer efeito significativo da educação sobre a escolha pelo tabagismo.

\section{Estratégia Empírica e Seleção Amostral}

Para a investigação do impacto das externalidades da alfabetização na probabilidade dos analfabetos serem fumantes, pode-se pensar que o status de ser fumante, F, é definido da seguinte forma:

$$
F_{i}=\left\{\begin{array}{l}
F_{1 i} \text { se } A N I_{i}=1 \\
F_{0 i} \text { se } A N I_{i}=0
\end{array}\right.
$$

$F_{1 i}$ é o status de fumante do indivíduo $i$ se este for $A N I$, e $F_{0 i}$ é o status de fumante do mesmo indivíduo $i$ se este não for $A N I$, ou equivalentemente $\operatorname{ser} A I$. 
O impacto de interesse sob o indivíduo $i$ é a diferença entre $F_{1 i}-F_{0 i}$, e o impacto médio para o grupo de $A N I$ é $E\left[F_{1 i}-F_{0 i} \mid A N I=1\right]$. Entretanto para um indivíduo $A N I$, ou para o grupo de $A N I$, não se consegue observar como seria o seu status referente ao consumo de tabaco caso não fosse $A N I$, ou seja, não se observa $F_{0 i}$ ou $E\left[F_{0 i} \mid A N I=1\right]$. Na ausência dessas informações poderse-ia pensar em utilizar a seguinte diferença de médias como estimativa para o impacto $E\left[F_{1 i} \mid A N I=1\right]-E\left[F_{0 i} \mid A N I=0\right]$. Essa medida só seria equivalente a $E\left[F_{1 i}-F_{0 i} \mid A N I=1\right]$, se o resultado médio da variável de interesse para o grupo ANI fosse exatamente o mesmo do $A I$ no caso em que os $A N I$ fossem $A I$, ou seja, $E\left[F_{0 i} \mid A N I=1\right]=E\left[F_{0 i} \mid A N I=0\right]$.

Entretanto, existe uma série de variáveis capazes de fazer com que $E\left[F_{0 i} \mid A N I=1\right] \neq E\left[F_{0 i} \mid A N I=0\right]$. Estes fatores podem fazer com que a diferença entre as médias dos dois distintos grupos contenha não apenas o impacto causal de interesse, mas também um componente de viés.

Primeiramente, para contornar essa questão, assume-se o pressuposto de que condicional às variáveis passíveis de observação, os resultados potenciais da variável de interesse (ser fumante ou não) são independentes do tratamento (ser $A N I$ ou $A I$ ). Formalmente $\left\{F_{1 i}, F_{0 i}\right\} \perp A N I_{i} \mid X_{i}$, em que $X_{i}$ é um conjunto de características observáveis do indivíduo $i$ capaz de influenciar a probabilidade ser fumante ou não.

Dessa forma, estimam-se diferentes especificações do seguinte modelo de probabilidade linear:

$$
F=\delta A N I+X \beta+\varepsilon
$$

Na equação (2) acima, F é um vetor ( $\mathrm{N}$ x 1) em que suas entradas são referentes ao status de fumo da amostra de analfabetos, assumindo valor 1 quando o indivíduo é fumante e zero quando não é; $A N I$ é uma variável binária que assume valor igual a 1 quando o indivíduo é $A N I$ e zero quando $A I ; \delta$ é o parâmetro de interesse que dá a magnitude da externalidade da alfabetização sobre a probabilidade de ser fumante; $\mathrm{X}$ é uma matriz de covariadas $(\mathrm{N} \times \mathrm{K}$ ) que podem influenciar o status de consumo de tabaco; $\beta$ é um vetor (K x 1) dos parâmetros das covariadas presentes em X; e $\varepsilon$ é o termo de erro que por hipótese se assume ter esperança condicional em $\mathrm{X}$ e em ANI igual a zero. Essa hipótese, se válida, garante que condicional às características observáveis dos indivíduos, o status de $A N I$ ou $A I$ é algo puramente aleatório, o que permite atribuir como externalidades da alfabetização a diferença de médias condicionais entre a probabilidade de ser fumante do grupo ANI e a probabilidade de ser fumante do grupo $A I$. Estimam-se diferentes especificações da equação (2), em que se incluem controles para a idade, gênero, 
cor, localização do domicílio, renda per capita domiciliar e características da composição familiar como domicílios com chefes homens e chefes homens solteiros.

É preciso dizer que, ao contrário do que impõe a equação (2), é bem possível que a probabilidade de uma pessoa ser fumante tenha uma relação não linear com as variáveis que a determinam. Dessa forma, o modelo especificado acima não seria o mais adequado, uma vez que, por exemplo, para qualquer idade de um indivíduo, uma variação na margem resulta no mesmo efeito sobre a probabilidade de ser fumante. Além disso, valores previstos para a probabilidade de ser fumante podem se situar fora do intervalo entre zero a um, o que não satisfaz a definição de probabilidade e tem consequência na estimação do desvio padrão do valor ajustado da variável de interesse.

Para contornar essas questões e garantir a robustez dos resultados obtidos, adotou-se como parte da estratégia empírica o modelo Probit. Tal modelo permite que a relação da variável binária dependente com as demais variáveis assuma uma forma não linear com valores restritos entre zero e um. Todas as especificações realizadas para o modelo de probabilidade linear também foram realizadas para o Probit.

No entanto, a identificação de externalidades da alfabetização sob a hipótese de seleção em observáveis é muito frágil. Tanto as estimações por MQO quanto as por Probit estão sujeitas a estarem viesadas em decorrência de fatores não observados relacionados com o status de analfabetismo não isolado dos indivíduos. Dessa forma, adotou-se o método de dois estágios com variáveis instrumentais cuja hipótese de identificação do impacto de interesse é de seleção em não-observáveis. A adequada implementação do método de variáveis instrumentais para a identificação do impacto requer as seguintes condições: i) a exogeneidade do instrumento, que no presente estudo significa que a probabilidade de ser ou não fumante não pode estar correlacionada com a variável instrumental; e ii) a correlação do instrumento com a variável de tratamento, ou seja, o instrumento deve afetar o perfil de alfabetização dos demais membros do domicilio.

Seguindo a orientação da literatura, utilizam-se duas variações exógenas de políticas de educação capazes de afetar as decisões de estudar dos indivíduos e, por consequência, a alfabetização. Uma delas é a lei 5.592 de 11 de agosto de 1971 e a outra é a Emenda Constitucional n. 14, de setembro de 1996. Em decorrência da primeira lei, o ensino de $1^{\circ}$ grau, englobando os antigos cursos primário e ginasial, passou a ser obrigatório, com duração de 8 anos e carga horária anual de 720 horas, destinando-se à formação de crianças e pré-adolescentes dos 7 aos 14 anos e eliminando a figura do exame de admissão para o ginasial. Já a Emenda Constitucional n. 14, de setembro de 1996 instituiu o Fundo de Manutenção e Desenvolvimento do Ensino Fundamental e de Valorização do Magistério (FUNDEF) que, a partir de 1998, reformulou o financiamento do ensino fundamental no Brasil ao vincular ao ensino funda- 
mental uma parcela dos recursos constitucionalmente destinados à educação ${ }^{3}$. Estas duas variações políticas são exógenas à decisão quanto ao consumo de tabaco dos indivíduos analfabetos e provavelmente exercem influência positiva sobre a decisão de tomar educação dos indivíduos, o que reflete na probabilidade de alfabetização dos mesmos. Recentemente no Brasil, Teixeira e Menezes-Filho (2012) também utilizaram mudanças de leis educacionais (lei 5.592) como instrumento para a escolaridade com a finalidade de medir o retorno privado da educação no mercado de trabalho.

A construção dos instrumentos é realizada da seguinte forma: cria-se uma variável binária com valor igual a 1 para aqueles indivíduos analfabetos que convivem com alguém alfabetizado que tinha menos de 14 anos em 1972 (a idade das pessoas que estavam no colégio na época da implementação da lei 5.592 e, portanto, foram afetados pela política), e zero caso contrário. Já para o instrumento associado ao Fundef, cria-se outra variável binária com valor igual a 1 para os indivíduos analfabetos que convivem com pessoas alfabetizadas que estavam em idade de frequentar a escola até a quarta série após 1998, ano em que o Fundef passou a vigorar, e zero caso contrário.

No primeiro estágio do método com variáveis instrumentais procede-se uma regressão da variável $A N I$ sobre os instrumentos e demais controles. No segundo estágio procede-se outra regressão com a variável $\mathrm{F}$ sobre a parte exógena da variável ANI (obtida no primeiro estágio) e demais controles.

Quanto à seleção amostral que será utilizada ela é obtida da Pesquisa PNAD de 2008, que contém uma pesquisa especial sobre tabagismo. A definição do status de alfabetização é obtida através da variável sabe ler e escrever. Aqueles que sabem são assinalados como alfabetizados, enquanto os que não sabem como analfabetos. Para a construção da variável de cor foram agrupados em "Brancos" os indivíduos declarados como brancos e amarelos devido à sua semelhança nos atributos sócio demográficos. Por fim, foi considerado fumante aquele indivíduo que consome algum produto de tabaco em frequência diária ou ocasional.

\section{Resultados}

A tabela 3 apresenta as estatísticas descritivas das variáveis que serão utilizadas. No total a seleção amostral contém 2878 analfabetos. Desta amostra as principais estatísticas são que $33.0 \%$ dos indivíduos são fumantes, $81.0 \%$ são $A N I, 26.0 \%$ são brancos e $53.0 \%$ são homens. Além disso, o fato de que 20.0\% dos analfabetos frequentaram escola também é interessante. A idade média dos analfabetos é elevada, 52.3 anos. Referente às estatísticas descritivas dos 
Instrumentos, $14.0 \%$ da amostra dos analfabetos convivem com alguém que é alfabetizado e estava em idade escolar quando ocorreu a implementação da lei 5.592. Já o percentual de analfabetos que convive com alguém que tinha idade escolar para ser afetado pelo Fundef é de 52.0\%.

TABELA 3: ESTATÍSTICAS DESCRITIVAS DAS VARIÁVEIS DE INTERESSE NA SELEÇÃO AMOSTRAL

\begin{tabular}{|c|c|c|c|c|c|}
\hline Variáveis & Obs & Média & $\begin{array}{c}\text { D e s v. } \\
\text { Pad. }\end{array}$ & Mín. & Máx. \\
\hline Fumante & 2878 & 0.33 & 0.47 & o & 1 \\
\hline ANI & 2878 & 0.81 & 0.39 & $\mathrm{O}$ & 1 \\
\hline Inst. Lei. 5.592 & 2878 & 0.14 & 0.35 & O & 1.00 \\
\hline Inst. Fundef & 2878 & 0.52 & 0.50 & O & 1.00 \\
\hline Idade & 2878 & 52.3 & 17.83 & 15 & 104 \\
\hline Branco & 2878 & 0.26 & 0.44 & $\mathrm{O}$ & 1 \\
\hline Homem & 2878 & 0.53 & 0.5 & O & 1 \\
\hline Setor Urbano & 2878 & 0.68 & 0.47 & o & 1 \\
\hline $\begin{array}{l}\ln (\text { Renda per } \\
\text { capita) }\end{array}$ & 2878 & $5 \cdot 72$ & 3.26 & 2.3 & 27.63 \\
\hline $\begin{array}{l}\text { Outros Fuman- } \\
\text { tes no Dom. }\end{array}$ & 2878 & 0.22 & 0.41 & $\mathrm{O}$ & 1 \\
\hline $\begin{array}{l}\text { Chefe Dom. } \\
\text { Homem }\end{array}$ & 2878 & 0.37 & 0.48 & O & 1 \\
\hline $\begin{array}{l}\text { Chefe Dom. Ho- } \\
\text { mem Solteiro }\end{array}$ & 2878 & 0.05 & 0.23 & $\mathrm{O}$ & 1 \\
\hline $\begin{array}{l}\text { Frequentou } \\
\text { Escola }\end{array}$ & 2878 & 0.2 & 0.4 & 0 & 1 \\
\hline
\end{tabular}

Fonte: Elaboração Própria

A Tabela 4 apresenta os resultados para as estimações do modelo de probabilidade linear e o modelo Probit. Para a primeira especificação que possui os controles para idade, cor, gênero, setor de localização do domicílio (urbano ou rural), renda per capita domiciliar, e presença de outros fumantes no domicílio foram obtidas estimativas de -12.1 pontos percentuais (p.p) e -15.3p.p para as externalidades da alfabetização sobre a probabilidade de ser fumante, todas significantes a 1.0\%. Estes resultados são semelhantes à especificação que inclui uma variável de controle para o caso em que o analfabeto é homem e chefe do domicílio. Já quando se controla pela possibilidade de que o analfabeto seja homem chefe de domicílio e solteiro, percebe-se que os coeficientes estimados são menores: entre -9.8p.p e -12.7p.p, mas ainda significantes a $1.0 \%$. 
Quando se inclui uma variável dummy que controla pela frequência escolar dos analfabetos no passado, constatou-se que os resultados se mantêm iguais tanto em termos de estimativas quanto em termos de significância.

TABELA 4: RESULTADO DAS ESTIMAÇÕES DOS MODELOS DE PROBABILIDADE LINEAR E PROBIT

Variável MQO1 MQO2 $\mathrm{MQO}_{3}$ MQO4 Probit1 Probit2 Probit3 Probit4 dep.

Fumante coef/dp coef/dp coef/dp coef/dp coef/dp coef/dp coef/dp coef/dp

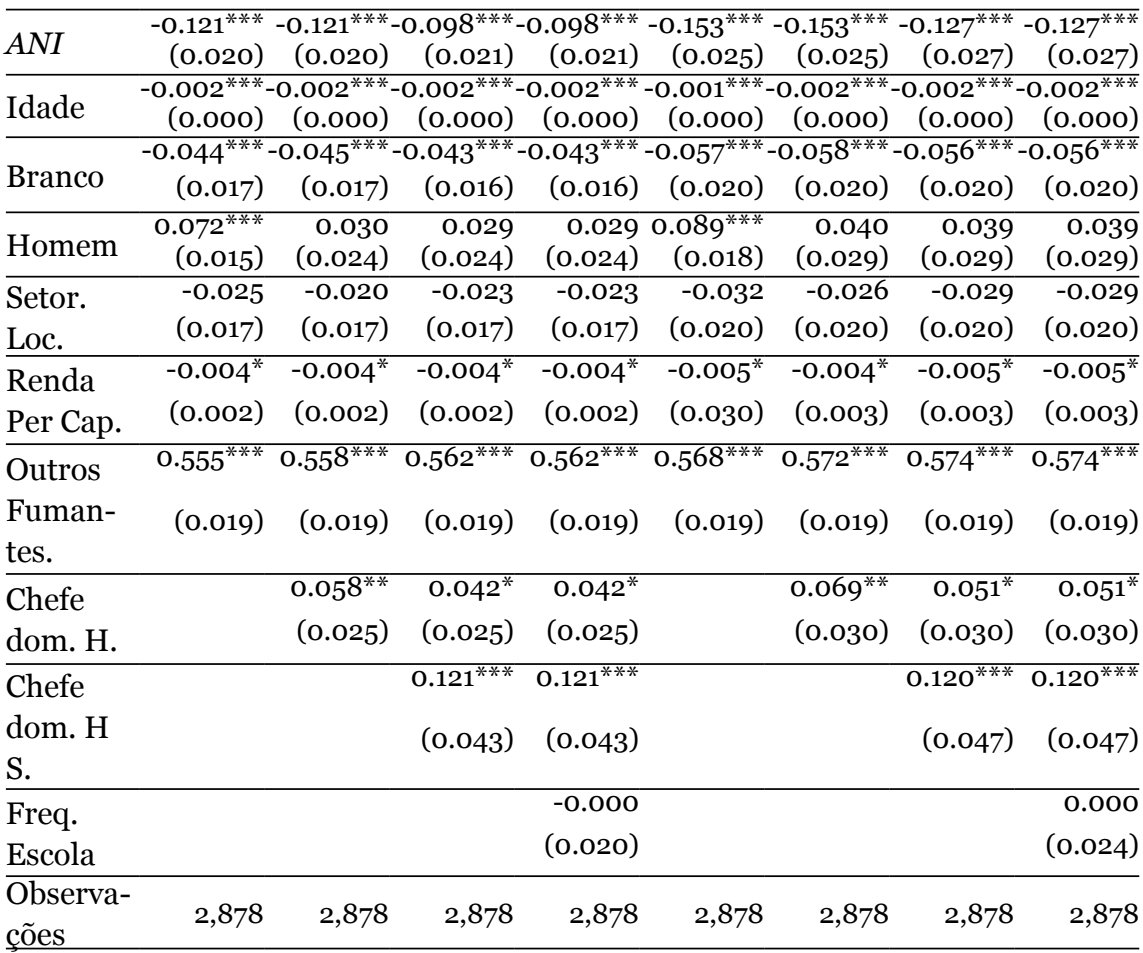

Nota: ${ }^{* * *} \mathrm{p}<0.01,{ }^{* *} \mathrm{p}<0.05,{ }^{*} \mathrm{p}<0.1$

Fonte: Elaboração Própria

A Tabela 5 apresenta os resultados do primeiro estágio das estimações do método de variáveis instrumentais em dois estágios. Percebe-se que, individualmente, os Instrumentos são significantes a 1.0\% e aumentam a probabilidade dos indivíduos serem ANI em todas as especificações. Os testes de validade dos instrumentos presentes na penúltima linha confirmam a validade conjunta dos mesmos. 
TABELA 5 : ESTIMAÇÕES DO PRIMEIRO ESTÁGIO DO MÉTODO DE VARIÁVEIS INSTRUMENTAIS

\begin{tabular}{|c|c|c|c|c|}
\hline Variável Dep. & VI_1 & VI_2 & VI_3 & VI_4 \\
\hline$A N I$ & coef/dp & coef/dp & coef/dp & coef/dp \\
\hline Inst. Lei. 5692 & $\begin{array}{r}0.164^{* * * *} \\
(0.011)\end{array}$ & $\begin{array}{r}0.163^{* * * *} \\
(0.011)\end{array}$ & $\begin{array}{r}0.144^{* * * *} \\
(0.011)\end{array}$ & $\begin{array}{r}0.144^{* * * *} \\
(0.011)\end{array}$ \\
\hline Inst. Fundef & $\begin{array}{r}0.371^{* * * *} \\
(0.013)\end{array}$ & $\begin{array}{r}0.371^{* * *} \\
(0.013)\end{array}$ & $\begin{array}{r}0.344^{* * *} \\
(0.013)\end{array}$ & $\begin{array}{r}0.344^{* * *} \\
(0.013)\end{array}$ \\
\hline Idade & $\begin{array}{r}-0.002^{* * * *} \\
(0.000)\end{array}$ & $\begin{array}{r}-0.002^{* * * *} \\
(0.000)\end{array}$ & $\begin{array}{r}-0.001^{* * * *} \\
(0.000)\end{array}$ & $\begin{array}{r}-0.001^{* * *} \\
(0.000)\end{array}$ \\
\hline Branco & $\begin{array}{r}0.024 \\
(0.015)\end{array}$ & $\begin{array}{r}0.024 \\
(0.015)\end{array}$ & $\begin{array}{r}0.018 \\
(0.014)\end{array}$ & $\begin{array}{r}0.018 \\
(0.014)\end{array}$ \\
\hline Homem & $\begin{array}{c}-0.003 \\
(0.013)\end{array}$ & $\begin{array}{r}0.003 \\
(0.018)\end{array}$ & $\begin{array}{r}0.009 \\
(0.018)\end{array}$ & $\begin{array}{r}0.009 \\
(0.018)\end{array}$ \\
\hline Setor Urbano & $\begin{array}{l}0.023^{*} \\
(0.014)\end{array}$ & $\begin{array}{r}0.022 \\
(0.014)\end{array}$ & $\begin{array}{l}0.033^{* *} \\
(0.013)\end{array}$ & $\begin{array}{r}0.032^{* *} \\
(0.013)\end{array}$ \\
\hline $\begin{array}{l}\ln (\text { Renda per } \\
\text { capita })\end{array}$ & $\begin{array}{l}-0.002 \\
(0.002)\end{array}$ & $\begin{array}{l}-0.002 \\
(0.002)\end{array}$ & $\begin{array}{l}-0.002 \\
(0.002)\end{array}$ & $\begin{array}{l}-0.002 \\
(0.002)\end{array}$ \\
\hline $\begin{array}{l}\text { Outros Fuman- } \\
\text { tes no Dom. }\end{array}$ & $\begin{array}{r}0.028^{* *} \\
(0.014)\end{array}$ & $\begin{array}{r}0.028^{* * *} \\
(0.014)\end{array}$ & $\begin{array}{r}0.011 \\
(0.014) \\
\end{array}$ & $\begin{array}{r}0.011 \\
(0.014) \\
\end{array}$ \\
\hline $\begin{array}{l}\text { Chefe Dom. } \\
\text { Homem }\end{array}$ & & $\begin{array}{l}-0.009 \\
(0.019)\end{array}$ & $\begin{array}{r}0.054^{* * * *} \\
(0.018)\end{array}$ & $\begin{array}{r}0.054^{* *-*} \\
(0.018)\end{array}$ \\
\hline $\begin{array}{l}\text { Chefe Dom. Ho- } \\
\text { mem Solteiro }\end{array}$ & & & $\begin{array}{r}-0.464^{* * *} \\
(0.031)\end{array}$ & $\begin{array}{r}-0.465^{* * *} \\
(0.030)\end{array}$ \\
\hline $\begin{array}{l}\text { Frequentou } \\
\text { Escola } \\
\end{array}$ & & & & $\begin{array}{r}0.012 \\
(0.015)\end{array}$ \\
\hline $\begin{array}{l}\text { Teste F de } \\
\text { Exclusão dos } \\
\text { Instrumentos }\end{array}$ & 441.42 & 441.18 & 369.17 & 369.42 \\
\hline Observações & 2,878 & 2,878 & 2,878 & 2,878 \\
\hline
\end{tabular}

Fonte: Elaboração Própria

A Tabela 6 apresenta as estimações do segundo estágio do método de variáveis instrumentais. Percebe-se que em todas as especificações as estimativas da magnitude das externalidades da alfabetização sobre a probabilidade de ser fumante são levemente menores do que as obtidas por mínimos quadrados ordinários e probit. Os coeficientes estimados se situam entre -9.5p.p e -7.op.p significantes a $5.0 \%$ e a $10.0 \%$. 
TABELA 6 : ESTIMAÇÕES DO SEGUNDO ESTÁGIO DO MÉTODO DE VARIÁVEIS INSTRUMENTAIS

\begin{tabular}{|c|c|c|c|c|}
\hline $\begin{array}{l}\text { Variável dep. } \\
\text { Fumante }\end{array}$ & $\begin{array}{l}\text { VI_1 } \\
\text { coef/dp }\end{array}$ & $\begin{array}{l}\text { VI_2 } \\
\text { coef/dp }\end{array}$ & $\begin{array}{c}\text { VI_3 } \\
\text { coef/dp }\end{array}$ & $\begin{array}{c}\mathrm{VI} / 4 \\
\text { coef/dp }\end{array}$ \\
\hline$A N I$ & $\begin{array}{l}-0.095^{* * *} \\
(0.038)\end{array}$ & $\begin{array}{l}-0.095^{* * *} \\
(0.038)\end{array}$ & $\begin{array}{l}-0.070^{*} \\
(0.042)\end{array}$ & $\begin{array}{l}-0.070^{*} \\
(0.042)\end{array}$ \\
\hline Idade & $\begin{array}{l}-0.001^{* * * *} \\
(0.000)\end{array}$ & $\begin{array}{l}-0.002^{* * * *} \\
(0.000)\end{array}$ & $\begin{array}{l}-0.002^{* * * *} \\
(0.000)\end{array}$ & $\begin{array}{l}-0.002^{* * * *} \\
(0.000)\end{array}$ \\
\hline Branco & $\begin{array}{l}-0.044^{* * * *} \\
(0.017)\end{array}$ & $\begin{array}{l}-0.045^{* * * *} \\
(0.016)\end{array}$ & $\begin{array}{l}-0.043^{* * * *} \\
(0.016)\end{array}$ & $\begin{array}{l}-0.043^{* * * *} \\
(0.016)\end{array}$ \\
\hline Homem & $\begin{array}{l}0.072^{* * * *} \\
(0.015)\end{array}$ & $\begin{array}{c}0.031 \\
(0.024)\end{array}$ & $\begin{array}{c}0.029 \\
(0.024)\end{array}$ & $\begin{array}{c}0.029 \\
(0.024)\end{array}$ \\
\hline Setor Urbano & $\begin{array}{l}-0.026 \\
(0.017)\end{array}$ & $\begin{array}{l}-0.021 \\
(0.017)\end{array}$ & $\begin{array}{l}-0.024 \\
(0.017)\end{array}$ & $\begin{array}{l}-0.024 \\
(0.017)\end{array}$ \\
\hline $\begin{array}{l}\text { In(Renda per } \\
\text { capita) }\end{array}$ & $\begin{array}{l}-0.004^{*} \\
(0.002)\end{array}$ & $\begin{array}{l}-0.004^{*} \\
(0.002)\end{array}$ & $\begin{array}{l}-0.004^{*} \\
(0.002)\end{array}$ & $\begin{array}{l}-0.004^{*} \\
(0.002)\end{array}$ \\
\hline $\begin{array}{l}\text { Outros Fumantes } \\
\text { no Dom. }\end{array}$ & $\begin{array}{l}0.553^{* * *} \\
(0.019)\end{array}$ & $\begin{array}{l}0.556^{* * *} \\
(0.019)\end{array}$ & $\begin{array}{l}0.560^{* * *} \\
(0.019)\end{array}$ & $\begin{array}{l}0.560^{* * *} \\
(0.019)\end{array}$ \\
\hline $\begin{array}{l}\text { Chefe Dom. Ho- } \\
\text { mem }\end{array}$ & & $\begin{array}{l}0.059^{* * *} \\
(0.025)\end{array}$ & $\begin{array}{c}0.040 \\
(0.025)\end{array}$ & $\begin{array}{c}0.040 \\
(0.025)\end{array}$ \\
\hline $\begin{array}{l}\text { Chefe Dom. Ho- } \\
\text { mem Solteiro }\end{array}$ & & & $\begin{array}{c}0.138^{* * * *} \\
(0.048)\end{array}$ & $\begin{array}{l}0.138^{* * *} \\
(0.048)\end{array}$ \\
\hline $\begin{array}{l}\text { Frequentou } \\
\text { Escola }\end{array}$ & & & & $\begin{array}{l}-0.000 \\
(0.020) \\
\end{array}$ \\
\hline Observações & 2,878 & 2,878 & 2,878 & 2,878 \\
\hline
\end{tabular}

Fonte: Elaboração Própria.

Para checar a robustez dos resultados apresentados nas tabelas 4 e 6, será utilizado um conceito alternativo de alfabetização dos indivíduos. Adota-se esse procedimento pelo fato de que a simples declaração quanto ao status de alfabetização pode não refletir a real capacidade de alguns indivíduos em ler e escrever. Em outras palavras, a variável que foi utilizada até então seria uma proxy para a alfabetização com erros de medida, o que viesaria as estimativas de interesse. Dessa forma, faz-se o seguinte refinamento à definição de alfabetização adotada até então: serão assinalados como alfabetizados aqueles indivíduos que se declaram alfabetizados, mas frequentaram escola pelo menos por quatro anos. Acredita-se que esse refinamento fornecerá uma melhor medida de alfabetização da população.

Com a nova definição foram realizadas estimações apenas por MQO e Variáveis Instrumentais. Os resultados das estimações do primeiro estágio do método de variáveis instrumentais foram omitidos devido à sua similaridade com as estimações apresentadas na Tabela 5 .

Os resultados das estimações por MQO e VI com a definição alternativa de alfabetização estão apresentados na Tabela 7. Em geral, os resultados obtidos são similares aos anteriores. Em termos de magnitude, para as duas primeiras especificações as estimativas de MQO estão levemente menores, passando de -12.op.p para algo em torno de -10.op.p (significantes a 1.0\%). Por variáveis 
instrumentais os resultados também são semelhantes, estimativas menores do que em MQO em torno de -9.0p.p e -7.4p.p significantes a 1.0\%.

TABELA 7: RESULTADO DAS ESTIMAÇÕES POR MQO E VI COM REFINAMENTO DA DEFINIÇÃO DE ALFABETIZAÇÃO

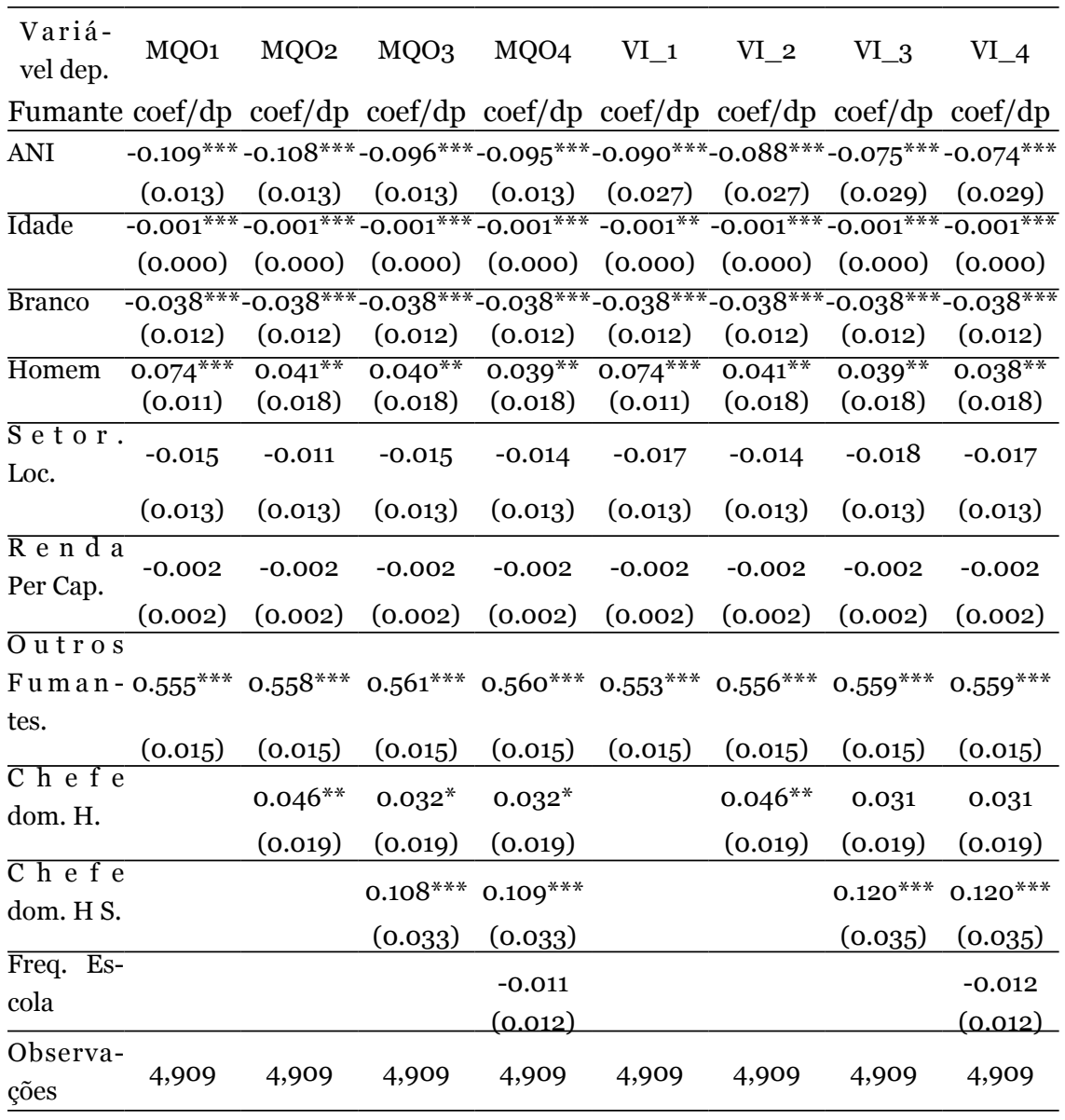

Nota: $* * * \mathrm{p}<0.01, * * \mathrm{p}<0.05, * \mathrm{p}<0.1$.

Fonte: Elaboração Própria

\section{Considerações Finais}

Este trabalho objetivou contribuir tanto com a literatura de economia da saúde que investiga os fatores determinantes do consumo de tabaco quanto à literatura de externalidades da alfabetização, uma vez que é o primeiro estudo que trata metodologicamente à questão da causalidade reversa na estimação da magnitude das externalidades da alfabetização através do uso de variáveis instrumentais. Os resultados obtidos indicam que o fato de haver alguém 
alfabetizado dentro de um domicílio acarreta em uma redução média entre 7.0\% e 9.5\% na probabilidade de indivíduos analfabetos serem fumantes. Os resultados são robustos às diferentes metodologias adotadas e às duas medidas de alfabetização utilizadas no trabalho.

Diante da constatação de que o Ceará é um dos estados que mais tem fumantes e Analfabetos Isolados, é provável que o elevado percentual de fumantes dentro da população cearense seja, entre outras coisas, uma decorrência da elevada concentração de analfabetos isolados no Estado, dado as evidências empíricas encontradas neste estudo. Dessa forma, as recomendações de políticas públicas são que políticas de alfabetização devem ser direcionadas para regiões e grupos demográficos dentro do Ceará em que a presença de analfabetismo isolado seja um traço marcante do perfil da população, pois dessa forma é possível que haja redução do percentual de fumantes. A longo prazo, os efeitos de tais políticas podem acarretar em poupança para o sistema único de saúde através da redução dos gastos com doenças associadas diretamente a epidemia tabágica.

\section{Referências}

Almeida-Duran, R. (2005). Proximate Literacy, Inter and Intra household Externalities and Child Health Outcomes: Evidence from India. Mimeo. Columbia University: New York, NY, 2005.

Basu, K. \& Foster, J. (1998). On Measuring Literacy, Economic Journal, vol. 108, p.p. 173-349, 1998.

Basu. K. \& Lee. T. (2008). A New and Easy-to-Use Measure of Literacy, Its Axiomatic Properties and an Application. Working Papers 08-04, Cornell University, Center for Analytic Economics.

Basu. K. Narayan., A. Narayan \& Ravallion. M. (2002). Is literacy shard within households? Theory and Evidence from Bangladesh, Journal of Labor Economics, vol. 8, p.p. 649-655, 2002.

Blunch, N., Verner D. (2000). Is Functional Literacy a Prerequisite for Entering the Labor Market?An Analysis of the Determinants of Adult Literacy and Earnings in Ghana. World Bank Working Paper 0o/05, 2000.

Borooah, V. K. (2002). The Role of Maternal Literacy in Reducing the Risk of Child Malnutrition in India, ICER Working Papers 31-2002, ICER - International Centre for Economic Research, 2002.

Chiswick, B. R., Repetto, C. (200o). Immigrant Adjustment in Israel: Literacy and Fluency in Hebrew and Earnings. Institute for the Study of Labor. Discussion Paper Series 177, 2000.

Cutler, D.M; Lleras-Muney, A. (2006). Education and health: evaluation theories and evidence. NBER Working Paper 12352, 2006. 
De Baldini Rocha, M. S., and Ponczek, V. P. (2011). The effects of adult literacy on earnings and employment, Economics of Education Review, 30, issue 4, p. 755-764, 2011.

Dutta, I. (2004). Generalized measures of literacy, Mathematical Social Sciences, 48: 68-90, 2004.

Elité, F.; Jones, A. (2011). Schooling and Smoking among the baby boomers: An evaluation of the impact of educational expansion in France. Journal of Health Economics, vol. 30, p. 811-831, 2011.

Farrell, P.; Fuchs, V.R. (1982). Schooling and health: the cigarette connection. Journal of Health Economics, n.1, p. 217-230, 1982

Fuchs, V.R. (2004). Reflections on the socio-economic correlates of health. Journal of Health Economics, vol. 23, p. 653-661, 2004.

Gibson. J. (2001). Literacy and Intra household Externalities, World Development, vol. 29, p.p. 155-166, 2001.

Green, D.A, Riddell, W.C. (2001). Labour Market Institutions and Outcomes: A Cross-National Study. Department of Economics McMaster University Discussion Paper 01-05, 2001.

Grossman, M. (2006). Education and nonmarket outcomes. In: Hanushek, E. Welch, F. (eds.) Handbook of the Economics of Education, vol.2, North-Holland, Amsterdam.

Grossman, M. (2000). The Human Capital Model. In: Culyer, A.J.; Newhouse, J.P. (eds.) Handbook of Health Economics, vol. 1A, Elsevier, Amsterdam, p. 347408, 2000.

INCA. Inca e Ministério da Saúde reafirmam combate ao tabagismo. http://www2. inca.gov.br/wps/wcm/connect/agencianoticias/site/home/noticias/2011/ inca_e_ministerio_da_saude_reafirmam_combate_ao_tabagismo. Acessado em 25 de agosto de 2011.

Iversen, V. ; Palmer-Jones, R. (2008). "Literacy Sharing, Assortative Mating, or What? Labour Market Advantages and Proximate Illiteracy Revisited," The Journal of Development Studies, Taylor and Francis Journals, vol. 44(6), pages 797-838, 2008.

Kemptner, D.; Jürges, H.; Reinhold, S. (2011). Changes in compulsoty schooling and the causal effect of education on health: Evidence from Germany. Journal of Health Economics, vol. 30, p. 340-354, 2011.

Lange, F. (2011). The role of education in complex health decisions: Evidence from cancer screening. Journal of Health Economics, vol. 30, p. 43-54, 2011.

Lee. T. (2008). Benchmarking the Effective Literacy Rate. Mathematical Social Sciences, vol 56, p.p $233-239,2008$.

Maddox. B. (2007). Worlds Apart? Ethnographic Reflections on "Effective Literacy" and Intrahousehold Externalities. World Development, vol. 35, p.p. 532-541, 2007.

Mishra S. (2001). "Isolated and Proximate Illiteracy," Economic and Political Weekly 36, 22,pp.2003-2008, 2001. 
Monteiro, C.A.; Cavalcante, T.M.; Moura, E.C.; Claro, R.M.; Szwarcwald, C.L. (2007). Population-based evidence of a strong decline in the prevalence of smokers in Brazil (1989-2003). Bull World Health Organ. Vol. 85, n. 7, p. 527-534, 2007.

Mukherjee. D. \& Gupta. M. R. (2006). Measures of Effective Literacy: A Theoretical and Empirical Note. Hitotsubashi Journal of Economics, vol. 47, p.p.17-35, 2006.

Pinto, M.; Ugá, M. Al. D. (2010). Os custos de doenças tabaco-relacionadas para o Sistema Único de Saúde. Cad. Saúde Pública, vol.26, no.6, p.1234-1245, Jun 2010.

Ribeiro, F. G e Cechin, L. A. W. (2012). As externalidades da alfabetização podem gerar uma porta de saída de curto prazo da pobreza para os beneficiários do Bolsa Família? Revista de Economia, vol. 38, no.2, p. 127-148, 2012.

Ribeiro, F. G e Souza, A.P.F. (2013). Uma investigação e aplicação das novas medidas de alfabetização no Brasil. Nova Economia, prestes a ser publicado, 2013.

Roemer, R. (1995). Acción legislative contra la epidemia mundial de tabaquismo. 2a edição. Genebra: Organización Mundial de la Salud, 1995.

Sander, W. Schooling and quitting smoking. (1995). Economics of Education Review, n.14, vol. 1, p. 23-33, 1995b.

Sander, W. (1995). Schooling and quitting smoking. Review of Economics and Statistics, vol.77, n.1, p. 191-199, 1995a.

Sarr, L. R. (2004). The Impact of Family Literacy on the Earnings of Illiterates: Evidence from Senegal. Cornell Food and Nutrition Policy Program Working Paper No. 159. Available at SSRN: http://ssrn.com/abstract=587381, 2004.

Subramanian, S. (2004). Measuring Literacy: Some Extensions of The Basu-Foster Framework. Journal of Development Economics, vol. 73, p.p. 453-463, 2004.

Teixeira, W. M. E Menezes-Filho, N. A. (2012). Estimando o Retorno à Educação do Brasil considerando a legislação educacional Brasileira como um instrumento. Revista de Economia Política, vol 32. no. 3, pp. 479-496, 2012.

Tenn, S; Herman, D; Wendling, B. (2010). The role of education in the production of health: An empirical analysis of smoking behavior. Journal of Health Economics, vol. 29, p.404-417, 2010.

Valentini. P. M. (2002). Should We Be Concerned About The Distribution of The Literacy Across Households? An Axiomatic Investigation. ”, CAE Working Paper \#02-15,. Available at: www.arts.cornell.edu/econ/cae/Valenti_Literacy. pdf, 2002.

World Health Organization (WHO). International Consultation on Tobacco and Youth: what in the world works? Singapore, 1999.

World Health Organization (WHO).The world health report 2002. Reducing risks, promoting healthy life. Geneva, 2002. 